\title{
Umbrales y prototipos: introducción al debate en torno a los estándares de prueba
}

\author{
Thresholds and Prototypes \\ An Introduction to the Debate on the Standards of Proof
}

Andrés Páez*

Recepción y evaluación de propuesta: 1/10/2016

Aceptación: 1/12/2016

Recepción y aceptación final: 4/7/2017

Resumen: Existen diversas perspectivas acerca de cómo se deben entender los estándares de prueba en el derecho. En el trabajo central de este número, Rodrigo Coloma sugiere que estos pueden ser entendidos como umbrales o como prototipos. Además, Coloma hace una taxonomía de los diferentes usos de los estándares de prueba que trasciende su simple función como distribuidores de errores. En sus réplicas al artículo, Raymundo Gama y Claudio Agüero hacen valiosas precisiones y serias críticas a esta forma de entender los estándares de prueba. Algunas de estas críticas dan en el blanco mientras que otras van dirigidas más a los presupuestos teóricos que a las tesis mismas de Coloma. En este artículo ofrezco un contexto más amplio para evaluar sus desacuerdos. Palabras clave: estándares de prueba, umbrales, prototipos, probabilidad subjetiva

Abstract: There are several approaches to the way in which standards of proof in law should be understood. In the central article in this issue, Ro-

* Departamento de Filosofía, Universidad de los Andes (Colombia).

Correo electrónico: apaez@uniandes.edu.co 
drigo Coloma suggests that they should be conceived either as thresholds either as prototypes. He also offers a taxonomy of the different uses of standards of proof, which transcends their function as error distributors. In their comments on the paper, Raymundo Gama and Claudio Agüero clarify and challenge this way of understanding standards of proof. Some of their criticisms are right on target, but many others are directed more at Coloma's theoretical presuppositions than at his substantial contributions. In this paper I offer a wider context to evaluate their disagreements.

Keywords: standards of proof, thresholds, prototypes, subjective probability

\section{Introducción}

El razonamiento probatorio en el derecho es una variedad del razonamiento inductivo, entendido este de la manera más general posible como razonamiento ampliativo. A partir de una serie de hechos básicos se formula una hipótesis que los trasciende. Los diferentes análisis del razonamiento inductivo se dividen en aquellos que poseen una regla de separación ${ }^{1}$ (detachment rule) y los que carecen de ella. A partir de los hechos recogidos empíricamente, dicha regla nos permite no solo formular una hipótesis sino también afirmar su verdad. Cuando existen varias hipótesis en competencia, la regla de separación también nos permite escoger una de ellas debido a que es la que cumple en el más alto grado alguna propiedad epistémica preestablecida. La regla requiere el uso de criterios, estándares o umbrales que nos indiquen el grado de apoyo que la información le debe proporcionar a la generalización o a la hipótesis para poder aceptarla como verdadera. Entre los análisis del razonamiento inductivo que cuentan con una regla de separación están la inducción por enumeración, la Inferencia a la Mejor Explicación ${ }^{2}$ y la teoría de la utilidad esperada ${ }^{3}$, mientras que el

1 La regla de separación de los sistemas inductivos no debe ser confundida con la llamada "ley de separación", nombre que es usado en algunos contextos para hacer referencia al modus ponens.

2 Lipton, P., Inference to the best explanation, segunda edición, London: Routledge, 2004.

3 Levi, I., The enterprise of knowledge, Cambridge: MIT Press, 1980; Hard choices, Cam- 
Umbrales y prototipos: introducción al debate en torno a los estándares de prueba

análisis bayesiano tradicional ${ }^{4}$ y la teoría de la confirmación de hipótesis de $\mathrm{Hempel}^{5}$ carecen de ella. Estas últimas no aceptan decisiones finales sobre la verdad de una hipótesis. Se limitan a rastrear los cambios en la relación de confirmación entre la hipótesis y la evidencia sin decirnos qué hacer o creer a medida que el apoyo proporcionado por la evidencia aumenta o disminuye.

Parece evidente que el razonamiento probatorio en el derecho debe corresponder al tipo de razonamiento inductivo que posee una regla de separación. Consideremos un par de ejemplos de aproximaciones teóricas que poseen una regla de este tipo. Pardo y Allen ${ }^{6}$ han adoptado la Inferencia a la Mejor Explicación como el modelo más adecuado para el razonamiento probatorio. En su opinión, la aceptación de una explicación potencial de los hechos del caso depende de criterios tales como la simplicidad, la consiliencia, la coherencia, entre otros. No hay una fórmula para combinar los criterios; más bien, "cada uno de estos criterios es un estándar que debe ser sopesado frente a los otros"7 a la luz de consideraciones teóricas y prácticas. Por su parte, Eleonora Cresto $^{8}$ ha combinado la Inferencia a la Mejor Explicación con funciones de utilidad epistémica para justificar las decisiones judiciales. Los estándares de prueba tradicionales son reinterpretados en términos de la utilidad esperada de la hipótesis aceptada sobre los hechos, frente a la utilidad de sus rivales. Cresto no se compromete con un umbral probabilístico único porque la utilidad epistémica y práctica de una

bridge: Cambridge University Press, 1985; Van Fraassen, B., Laws and Symmetry, Oxford: Clarendon Press, 1989; Maher, P., Betting on theories, Cambridge: Cambridge University Press, 1993.

4 Howson, C., Urbach, P., Scientific reasoning: the Bayesian approach, Chicago: Open Court, 1993; Earman, J., Bayes or bust? A critical examination of bayesian confirmation theory, Cambridge: MIT Press, 1992.

5 Hempel, C. G., "Studies in the logic of confirmation", Mind, 1945, p. 54, 1-26, 97-121.

6 Pardo, M. S., Allen, R. J. "Juridical proof and the best explanation", Law and Philosophy, 2008, p. 27, 223-268.

7 Ibid., p. 230.

8 Cresto, E., "Una teoría de la prueba para el ámbito jurídico: probabilidades inciertas, decisiones y explicación”, en Páez, A. (ed.), Hechos, evidencia y estándares de prueba, Bogotá: Ediciones Uniandes, 2015, pp. 89-119. 
hipótesis es altamente dependiente del contexto probatorio. En ambas aproximaciones es claro que existen criterios, umbrales y estándares para dar por verdadera una hipótesis sobre los hechos, aunque estos no son absolutos - como lo serían, por ejemplo, en el caso de la prueba clínica de un medicamento - sino altamente sensibles a las particularidades del contexto probatorio.

Ambos análisis del razonamiento probatorio, sin embargo, están enmarcados en la tradición teórica que se desarrolló en torno al common law. Cuando examinamos lo que ha ocurrido en el derecho continental, la situación es bastante diferente. Durante mucho tiempo este operó como si la relación de confirmación entre las pruebas y la hipótesis de culpabilidad o responsabilidad en un proceso tuviera un carácter absoluto y como si el juez tuviera la capacidad intuitiva de detectar la existencia o no de dicha confirmación. No existía la conciencia de que la confirmación de una hipótesis es una cuestión de grado, ni se tenía en cuenta que hay factores epistémicos, como el poder explicativo, que pueden ser integrados a las virtudes que buscamos en las hipótesis que aceptamos en un proceso judicial.

Esta situación ha comenzado a cambiar durante los últimos años como lo revela la larga lista de libros y artículos recientes sobre el tema que nos presenta Claudio Agüero en su contribución a este número. A este "boom", como lo llama Agüero, de literatura sobre razonamiento probatorio en español se suma ahora el artículo "Los usos de los estándares de prueba", de Rodrigo Coloma. Consciente de que este nuevo enfoque en la filosofía del derecho aún tiene mucho trecho por recorrer, Coloma arguye que el bajo nivel de influencia que han ejercido los estándares de prueba en el razonamiento probatorio se debe, por una parte, a que los teóricos no han clarificado suficientemente los aspectos más importantes de sus usos, y por otra, a que existe un escepticismo, derivado de las críticas de Laudan ${ }^{9}$, en torno a la posibilidad de alcanzar estándares de prueba objetivos y precisos. Su objetivo, en consecuencia, es contribuir a la clarificación del sentido del concepto 
Umbrales y prototipos: introducción al debate en torno a los estándares de prueba

y mostrar que su uso y su importancia no se limitan a la distribución de errores en las decisiones probatorias, como parecen creerlo muchos teóricos que se ocupan del tema.

En este escrito presentaré las tesis principales de Coloma y algunas de las críticas de Gama y Agüero. Parte de mi objetivo es sopesar y matizar dichas críticas para poder ofrecer un panorama más equilibrado de las propuestas de Coloma y del contexto teórico en el que son presentadas.

\section{El punto de partida: el lenguaje ordinario}

El punto de partida de Coloma es el análisis del uso del concepto de estándar en el lenguaje ordinario. A su juicio, este análisis puede ayudar a clarificar el sentido de "estándar de prueba" en el derecho. Coloma distingue dos sentidos predominantes: los estándares como umbrales y como prototipos. En el primer sentido, un estándar tiene un sentido puramente cuantitativo. Define un valor mínimo que se debe alcanzar para poder pertenecer a una categoría. Así, por ejemplo, uno de los estándares para clasificar un aceite de oliva como extra virgen es que tenga un nivel de acidez inferior a 1\%; y el estándar para que un licor sea considerado tequila es que debe contener al menos $51 \%$ de agave azul. El segundo sentido coloquial de "estándar" es utilizado cuando queremos comparar un ejemplar no clasificado con una muestra, modelo o patrón. Coloma utiliza el ejemplo de la calidad de un libro frente al cual la calidad de todos los demás libros debe ser juzgada. Este uso requiere de un juicio de semejanza que es puramente cualitativo.

Coloma no afirma que no existan más usos comunes del término. Se me ocurre, por ejemplo, un uso que combina los dos sentidos anteriores: el famoso metro estándar de platino localizado en las afueras de París, y que Wittgenstein volvió famoso en las Investigaciones filosóficas. Es un estándar cuantitativo, pero a la vez es un patrón o modelo. Existen además otros usos, señalados por Raymundo Gama en su comentario al artículo de Coloma, que se acercan al concepto de umbral sin ser cuantitativos. Por ejemplo, el estándar de calidad de vida tiene en 
cuenta factores no numéricos como el nivel de desarrollo personal, el grado de goce de derechos, entre otros. Los pares umbral-cualitativo y prototipo-cualitativo no parecen, por tanto, ser categorías enteramente independientes.

Aparte de las precisiones que se pueden hacer acerca del uso del término "estándar" en el lenguaje ordinario, es muy pertinente la pregunta que hace Gama acerca de la utilidad de comenzar el análisis de un concepto técnico como el de estándar de prueba en el derecho con una taxonomía de su uso no técnico. No hay ninguna garantía previa de que el sentido en el que es usada una expresión en un contexto se pueda trasladar automáticamente a otro. Yo añadiría un problema adicional. Ya sea que se entienda como umbral o como prototipo, el concepto de estándar en el uso cotidiano asume la existencia de criterios preexistentes bien definidos a partir de los cuales se hace la medición o la comparación de similitud. El contenido de agave azul, el grado de acidez, e incluso la calidad de un texto a juicio de los expertos son criterios con los que se cuenta de antemano. En contraste, en el caso de los estándares de prueba en el derecho lo que está en juego es justamente la definición de los criterios apropiados para darle contenido a dicho concepto. El desacuerdo teórico acerca de la mejor manera de analizar el razonamiento inductivo, y acerca de cómo incluir el razonamiento probatorio dentro de alguno de los modelos inductivos, es en gran parte un desacuerdo acerca de cuáles deben ser los criterios que rigen la aplicación de la regla de separación mencionada al comienzo. ¿Debemos usar el carácter explicativo como criterio para escoger una hipótesis? ¿O quizás utilizar un filtro numérico que represente la probabilidad subjetiva del determinador de los hechos? ¿O quizás la utilidad epistémica de la hipótesis? Es necesario responder estas preguntas antes de poder establecer puentes entre el concepto técnico y el concepto coloquial. En otras palabras, si queremos entender el problemático concepto de estándar de prueba en el derecho, quizás no sea buena idea comparar contextos en donde la aplicación de estándares ya cuenta con criterios y protocolos bien definidos con otros en los que no existe un consenso acerca de cuáles son los criterios más adecuados. 
Umbrales y prototipos: introducción al debate en torno a los estándares de prueba

\section{III. ¿Umbrales cuantitativos?}

Agüero señala un problema adicional acerca del uso no jurídico de la palabra "umbral". El término denota un límite, el valor mínimo de una magnitud. Al ser un sentido importado de contextos científicos y técnicos, viene cargado con el presupuesto de que en el contexto probatorio en el derecho también va a ser posible cuantificar, medir la magnitud de alguna propiedad otorgada a una hipótesis por las pruebas. En su opinión, sin embargo, el término "umbral" solo puede ser usado metafóricamente, y la metáfora no es suficiente para llenar el vacío conceptual detectado inicialmente por Coloma. Lo mismo ocurre, según Agüero, con el uso corriente de la palabra "prototipo". La palabra denota un ejemplar perfecto, un patrón cuyas medidas sirven de base para la elaboración de objetos semejantes. Pero para que la semejanza sea efectiva, es necesario, una vez más, poseer magnitudes precisas, cantidades medibles, y es esto justamente lo que se pone en duda en el contexto probatorio.

Agüero no ofrece una alternativa no metafórica para entender y explicar la relación entre los medios de prueba y la aceptación de un hecho probado. Su intención en este escrito es llamar poderosamente la atención acerca de la fragilidad conceptual de las discusiones en torno a los estándares de prueba. Me parece, sin embargo, que es necesario señalar que las rupturas entre el razonamiento jurídico, el científico e incluso el cotidiano no son tan profundas como lo sugiere Agüero. Como lo indiqué al comienzo, el razonamiento inductivo hace parte fundamental de cualquier actividad donde sea necesario comprometerse con una hipótesis y tomar decisiones en situaciones de incertidumbre. Existe una continuidad entre todos estos contextos. El problema no es que no existan conceptos para entender cómo funciona el razonamiento inductivo, ni tampoco que no sea posible pensar en magnitudes o grados de prueba. Tanto los grados de creencia como las utilidades epistémicas son ejemplos de formas de conceptualizar el razonamiento inductivo cuantitativamente. El problema de fondo es más bien determinar cuál de las teorías preexistentes acerca del razonamiento inductivo se ajusta mejor a las exigencias del ejercicio pro- 
batorio en el derecho. Es válido que a Agüero no lo convenza ninguna de las teorías inductivas cuantitativas existentes, pero esa discusión trasciende los límites del derecho y se tiene que plantear como una objeción epistemológica mucho más general.

Ahora bien, cabe preguntarse si Coloma está realmente comprometido con una concepción cuantitativa de los estándares de prueba al plantear la posibilidad de concebirlos como umbrales. Ni Gama ni Agüero se atreven a atribuir esta posición a Coloma. Para Gama, Coloma "parece titubear con la posibilidad de emplear el cálculo numérico de probabilidades". Si bien Coloma encuentra problemas en su aplicación, al mismo tiempo el uso mismo del concepto parece presuponer un quantum mínimo de probabilidad. Al respecto afirma Gama: "En relación con este punto, me parece que no hay margen para titubear al respecto pues nos encontramos ante una disyuntiva que no admite matices". Ante la disyuntiva, Gama claramente adopta una posición de rechazo frente al empleo del cálculo de probabilidades y respalda su posición con el aparente consenso que existe entre muchos de los autores más conocidos en Hispanoamérica (Allen, Haack, Gascón, Ferrer, Bayón, entre otros) acerca de la inadecuación del cálculo de probabilidades para entender el estándar de prueba. El argumento no parece muy convincente, pues aunque en nuestro medio son más los que rechazan que los que aceptan una concepción probabilística de los umbrales de prueba, en otras latitudes dicha tradición persiste y goza de buena salud ${ }^{10}$.

Agüero, por su parte, describe la posición de Coloma como la de un "optimista epistémico desconfiado". Su desconfianza radica justamente en no tener demasiada fe en las herramientas conceptuales que

10 Para una revisión de la literatura más reciente sobre el uso de herramientas estadísticas y bayesianas en el razonamiento probatorio, véase Fenton, N., Neil, M. y Lagnado, D., "A general structure for legal arguments about evidence using Bayesian networks", Cognitive Science, 2013, p. 37, 61-102; Fenton, N., Neil, M. y Berger, D., "Bayes and the law", Annual Review of Statistics and Its Application, 2016, p. 3, 51-77. En español se puede consultar Lillquist, E., "Teoría de la utilidad esperada y variabilidad en el estándar de prueba más allá de toda duda razonable", en Cruz Parcero, J. A. y Laudan, L. (eds.), Prueba y estándares de prueba en el derecho, México: UNAM, 2010, pp. 141-165; Páez, A., "Estándares múltiples de prueba en medicina y derecho", en Páez, A., (ed.), Hechos, evidencia y estándares de prueba, Bogotá: Ediciones Uniandes, 2015, pp. 123-152 y Cresto, E., op. cit. 
utiliza para dilucidar qué es un estándar de prueba. Es decir, Agüero tampoco logra identificar un compromiso evidente por parte de Coloma con el uso del cálculo de probabilidades. Al igual que Gama, Agüero rechaza ese uso. Su argumentación hace una ingeniosa comparación entre la invención de las escalas cuantitativas para medir la temperatura y la formulación de un estándar cuantitativo en el derecho con el fin de sacar a la luz las diferencias esenciales que existen entre los dos casos. Una vez más, Agüero expresa su incredulidad de que sea posible generar un "termómetro probatorio" que mida alguna magnitud cuantificable. Sin embargo, la comparación adecuada no es realmente entre el contexto científico y el jurídico; es más bien entre el científico y el epistemológico, porque el "termómetro probatorio" tendría que funcionar en cualquier contexto en el que se use el razonamiento inductivo, no solamente en el jurídico.

A mi modo de ver, Coloma ni titubea ni desconfía. Su propósito es plantear posibilidades de interpretación y mostrar las implicaciones de cada una de esas posibilidades. Así, por ejemplo, al final del artículo Coloma muestra las consecuencias jurídicas de entender los estándares como umbrales o como prototipos, sin que esto implique un titubeo o una falta de compromiso con alguna tesis sustancial. En cuanto a su compromiso o no con el cálculo de probabilidades, es claro que la manera más común de asignarle contenido a la noción de umbral es concibiéndolo como un límite cuantitativo. Un umbral implica un "valor mínimo", nos dice Coloma, y "la posibilidad de construir escalas de medición". Y más adelante añade que "el cálculo probabilístico, pese a todas sus dificultades, resulta prometedor para esta perspectiva". Coloma no ofrece detalles de cómo se debe aplicar el cálculo de probabilidades a la concepción de estándar como umbral, pero creo que deja claro que admite esa posibilidad. 


\section{Los usos de los estándares de prueba}

La contribución de Coloma no se limita a clarificar el sentido de "estándar de prueba". El cuerpo central del artículo está dedicado a hacerle ver al lector que el uso de estándares de prueba, especialmente en el civil law, tiene consecuencias e implicaciones que no son nada evidentes, pero que tienen una importancia capital dentro de los procesos judiciales. Generalmente, se afirma que los estándares de prueba sirven esencialmente para determinar la distribución de errores en los procesos judiciales. Un estándar muy alto generará muchos falsos negativos, mientras que un estándar muy bajo tendrá el efecto contrario. La fijación del estándar de prueba sirve entonces para decidir la proporción de falsos positivos y falsos negativos que una sociedad está dispuesta a aceptar. Coloma nos muestra que limitar el uso de los estándares de prueba a ese fin es empobrecer el concepto. A continuación discutiré algunos de los usos señalados por Coloma.

Los usos de los estándares de prueba son clasificados por Coloma en tres grandes modalidades: i) la determinación de la cantidad de errores esperables del sistema de adjudicación, II) la distribución entre las partes de los errores que produce el sistema, y iii) la forma que adoptan los hechos probados.

La primera modalidad corresponde al uso más comúnmente aceptado de los estándares de prueba mencionado anteriormente: la distribución general de errores. Coloma incluye bajo esta primera categoría la influencia que tiene el estándar de prueba en el momento de decidir si es conveniente participar, o retirarse anticipadamente de un litigio. Las partes hacen un cálculo de sus probabilidades de éxito a partir del estándar utilizado, y deciden si es conveniente proseguir. El estándar también sirve como guía para que las partes decidan cuánta prueba es necesario producir sin incurrir en costos excesivos y desproporcionados. Finalmente, Coloma también incluye aquí la influencia que puede tener el estándar sobre la clase de inferencias que es válido realizar, sobre "los saltos argumentales que resultan tolerables". Sin embargo, como señala Gama, no es clara la relación entre el estándar de prueba y el estándar argumentativo, por llamarlo de alguna manera. ¿Por qué pensar que un 
Umbrales y prototipos: introducción al debate en torno a los estándares de prueba

estándar de prueba bajo me otorga una cierta licencia argumentativa? Es cierto que si el estándar de prueba es, por ejemplo, la preponderancia de la prueba, las pruebas solo deberán otorgarle a la hipótesis un grado de confirmación superior al $50 \%$, pero eso no significa que para llegar a ese grado de confirmación sea admisible violar reglas lógicas o argumentativas. Como afirma Gama, "el hecho de que haya un salto argumental en alguno de los tramos de la inferencia convertiría a esta en inaceptable". En síntesis, creo que existe una confusión entre la firmeza de la hipótesis y la firmeza de los argumentos que fijan ese grado de firmeza. Asociado con lo anterior, también resulta problemática la afirmación de Coloma según la cual un estándar de prueba exigente "favorece que las decisiones adoptadas por los tribunales de justicia sean, en términos generales, más fiables que las que resultan de la utilización de estándares de prueba menos exigentes". Un tribunal civil que se rija por un estándar de prueba bajo, como la preponderancia de la prueba, puede ser altamente fiable en las decisiones que toma debido, por ejemplo, a las bondades del diseño institucional. No existe ningún tipo de incompatibilidad entre las dos cosas.

La segunda modalidad de uso de los estándares de prueba consiste en la distribución entre las partes de los errores que produce el sistema. Aquí se incluye la distribución entre las partes de falsos positivos y falsos negativos. Coloma, al igual que la gran mayoría de los teóricos, es un poco escéptico acerca de este uso de los estándares de prueba debido a nuestra ignorancia acerca de la distribución real de errores. Otro uso que cae bajo esta modalidad es el de determinar los opuestos que compiten en la decisión probatoria. Los opuestos a los que se refiere Coloma son: i) dar por probado/no probado un hecho $x$, y ii) dar por probado un hecho $x$ o su contrario. Si el estándar de prueba es muy alto, se trata del primer caso. Cuando el estándar no es alcanzado, simplemente se declara como no probado el hecho en cuestión. Pero cuando el estándar es la preponderancia de la prueba, se trata del segundo caso, pues la no prueba del hecho $x$ implica la prueba de su contrario.

La última modalidad de uso de los estándares de prueba es la de determinar la forma de los hechos probados. Por una parte, Coloma afirma que el estándar influye en la clase de razonamiento que adoptan 
las partes y los jueces. Este puede ser atomista u holista. En el primer caso se divide la hipótesis a probar en sus proposiciones constitutivas y estas se ponen a prueba independientemente, mientras que en el segundo caso se analiza la hipótesis globalmente sin entrar a considerar individualmente la fortaleza de sus componentes. Según Coloma, si el estándar es muy alto, por ejemplo, si es "más allá de toda duda razonable", se favorece la primera aproximación porque cada proposición debe cumplir dicho estándar. La que no lo haga dará pie para una duda razonable. Gama objeta que aquí ocurre algo similar a lo que se señaló en el caso de la relación entre el estándar de prueba y la calidad de la argumentación. Así el estándar sea muy bajo, la existencia de una proposición atómica implausible o increíble tiene un efecto devastador sobre la hipótesis general. La fortaleza de las demás proposiciones no puede apalancar proposiciones carentes de todo sustento. Además, como señala Gama, no es claro que el órgano decisor en realidad tenga la opción de adoptar un enfoque $u$ otro. La relación entre ambos enfoques es un asunto que ha sido discutido en la literatura y la pregunta es si los estándares de racionalidad del derecho probatorio permiten abandonar el enfoque atomista ${ }^{11}$.

El segundo uso de los estándares de prueba que cae bajo esta última modalidad es el de determinar el estatus doxástico de los hechos probados. Estos pueden ser concebidos o bien como creencias o bien como la aceptación de un proceso de validación. En este caso la correspondencia sería entre el cumplimiento de un estándar de prueba alto y la generación de creencias en el determinador de los hechos, y un estándar bajo y la mera aceptación del cumplimiento de los requisitos legales. Coloma deja apenas planteado el asunto, sobre el que, por lo demás, existe una amplia literatura y una falta notoria de consenso. Faltaría aclarar qué se entiende exactamente por aceptación, por creencia, y además lidiar con la diferencia entre una concepción filosófica y una psicológica de la creencia. Hay aquí, pues, una veta lista para ser explotada en futuros trabajos.

11 Véase Accatino, D., "Atomismo y holismo en la justificación probatoria", Isonomía, 40, 2014, pp. 17-59. 
Umbrales y prototipos: introducción al debate en torno a los estándares de prueba

\section{Consideraciones finales}

El artículo de Coloma termina con un interesante ejercicio en el que se analizan los diferentes pasos del razonamiento probatorio teniendo en cuenta si se trata de un estándar alto o bajo. El tipo de estándar determina, a su vez, el tipo de razonamiento, la forma de los hechos, la actitud doxástica, etc., según lo que se explicó en el apartado anterior. El análisis también toma en cuenta si el estándar es entendido como umbral o como prototipo. No entraré a analizar en detalle los pasos explicados por Coloma. Me interesa, más bien, terminar con una breve reflexión sobre la idea de prototipo, que es un concepto sobre el que hubiera sido deseable mayor claridad y profundidad.

Un primer problema al que se enfrenta esta forma de concebir el estándar de prueba es que no es en absoluto claro qué es lo que estamos buscando cuando queremos adoptar un prototipo. ¿Se trata de una decisión judicial que posea virtudes argumentativas, procesales y probatorias que la hagan sobresaliente? En tal caso, ¿qué características y virtudes debe tener? ¿Cuál debe ser la relación entre las pruebas y el hecho a probar? Dado que existen límites a la posibilidad de generalizar un caso particular, ¿qué criterios se deben seguir para no excedernos en dichas generalizaciones? Y si solo tomamos en cuenta las características más generales del prototipo, ¿no se corre el riesgo de que este resulte inservible como base para tomar una decisión?

En los sistemas que utilizan jurados se ha detectado que estos utilizan sus propios prototipos o esquemas de un crimen a la hora de juzgar la culpabilidad del imputado. Así, el acusado es juzgado culpable por un miembro del jurado cuando existe un traslape significativo entre las circunstancias del crimen y el caso prototípico que tiene en su cabeza ${ }^{12}$. No hay razón para pensar que algo similar no ocurra en los sistemas continentales en los que el juez se puede haber formado, como resultado de su práctica profesional, un prototipo altamente subjetivo de

12 Ogloff, J. R. P., "Jury decision making and the insanity defense", en Castellan, N. J., (ed.), Individual and group decision making. Current issues, Hillsdale: Lawrence Earlbaum, 1993, pp. 167-202. 
cada tipo de crimen. Si lo que se pretende al formular el estándar de prueba en términos de prototipos es eliminar el uso de probabilidades subjetivas, hay que tener en cuenta que los prototipos individuales y subjetivos también entran en juego en el momento de la decisión.

Finalmente, si existe un continuo en el razonamiento inductivo en la vida cotidiana, en la ciencia y en el derecho, como lo he planteado en esta introducción, para darle contenido a la idea de estándar de prueba debemos buscar aquellos elementos en común al razonamiento en todas estas esferas. La idea de umbral parece mucho más cercana a una práctica que se puede identificar en todas ellas. En cambio, la idea de prototipo solo es utilizada en contextos muy específicos y es menos propicia a la generalización. Es quizás por esta razón que la idea de estándar de prueba como umbral se niega a desaparecer de la literatura sobre razonamiento probatorio.

\section{Bibliografía}

Accatino, D., "Atomismo y holismo en la justificación probatoria", Isonomía, 40, 2014, pp. 17-59.

Cresto, E., "Una teoría de la prueba para el ámbito jurídico: probabilidades inciertas, decisiones y explicación", en Páez, A. (ed.), Hechos, evidencia y estándares de prueba, Bogotá: Ediciones Uniandes, 2015, pp. 89-119.

Earman, J., Bayes or bust? A critical examination of bayesian confirmation theory, Cambridge: MIT Press, 1992.

Fenton, N., Neil, M. y Lagnado, D., "A general structure for legal arguments about evidence using Bayesian networks", Cognitive Science, 2013, p. 37, 61-102.

Fenton, N., Neil, M. y Berger, D., "Bayes and the law", Annual Review of Statistics and Its Application, 2016, p. 3, 51-77.

Hempel, C. G., "Studies in the logic of confirmation", Mind, 1945, p. $54,1-26,97-121$.

Howson, C. y Urbach, P., Scientific reasoning: the Bayesian approach, Chicago: Open Court, 1993. 
Umbrales y prototipos: introducción al debate en torno a los estándares de prueba

Laudan, L., Truth, error and criminal law: An essay in legal epistemology, Cambridge: Cambridge University Press, 2006.

Levi, I., The enterprise of knowledge, Cambridge: MIT Press, 1980.

Levi, I., Hard choices, Cambridge: Cambridge University Press, 1985.

Lillquist, E., "Teoría de la utilidad esperada y variabilidad en el estándar de prueba más allá de toda duda razonable", en Cruz Parcero, J. A. y Laudan, L. (eds.), Prueba y estándares de prueba en el derecho, México: UNAM, 2010, pp. 141-165

Lipton, P., Inference to the best explanation, segunda edición, London: Routledge, 2004.

Maher, P., Betting on theories, Cambridge: Cambridge University Press, 1993.

Ogloff, J. R. P., "Jury decision making and the insanity defense", en Castellan, N. J. (ed.), Individual and group decision making. Current issues, Hillsdale: Lawrence Earlbaum, 1993, pp. 167-202.

Páez, A., "Estándares múltiples de prueba en medicina y derecho", en Páez, A. (ed.), Hechos, evidencia y estándares de prueba, Bogotá: Ediciones Uniandes. 2015, pp. 123-152.

Pardo, M. S. y Allen, R. J., "Juridical proof and the best explanation", Law and Philosophy, 2008, p. 27, 223-268.

Van Fraassen, B., Laws and Symmetry, Oxford: Clarendon Press, 1989. 